\title{
Annihilation vs. Decay: Constraining Dark Matter Properties from a Gamma-Ray Detection in Dwarf Galaxies
}

\author{
Sergio Palomares-Ruiz ${ }^{* \dagger}$ \\ Centro de Física Teórica de Partículas (CFTP), \\ Instituto Superior Técnico, \\ Avenida Rovisco Pais 1, 1049-001 Lisboa, Portugal \\ E-mail: sergio.palomares.ruiz@ist.utl.pt \\ Jennifer M. Siegal-Gaskins \\ Center for Cosmology and Astro-Particle Physics (CCAPP), \\ The Ohio State University, \\ 191 W. Woodruff Ave., Columbus, OH 43210 USA \\ E-mail: jsg@mps.ohio-state.edu
}

\begin{abstract}
Although most proposed dark matter candidates are stable, in order for dark matter to be present today, the only requirement is that its lifetime is longer than the age of the Universe, $t_{U} \simeq 4 \times$ $10^{17} \mathrm{~s}$. Moreover, the dark matter particle could be produced via non-thermal processes and have a larger annihilation cross section from the canonical value for thermal dark matter, $\langle\sigma v\rangle \sim$ $3 \times 10^{-26} \mathrm{~cm}^{3} \mathrm{~s}^{-1}$. We propose a strategy to distinguish between dark matter annihilation and/or decay in the case that a clear signal is detected in future gamma-ray observations of Milky Way dwarf galaxies with gamma-ray experiments. The discrimination between these cases would not be possible in the case of the measurement of only the energy spectrum. We show that by studying the dependence of the intensity and energy spectrum on the angular distribution of the signal, the origin of the signal could be identified, and some information about the presence of substructure might be extracted.
\end{abstract}

Identification of Dark Matter 2010-IDM2010

July 26-30, 2010

Montpellier France

\footnotetext{
* Speaker.

$\dagger$ This work was partially supported by the Portuguese FCT through CERN/FP/109305/2009 and CFTP-FCT UNIT 777, which are partially funded through POCTI (FEDER), and by the Spanish Grant FPA2008-02878 of the MICINN.
} 


\section{Introduction}

The existence of dark matter (DM) is inferred from many different astrophysical and cosmological observations, which indicate that it constitutes about $80 \%$ of the mass content of the Universe. However, aside from its gravitational interactions, very little is known about its nature. Among the many proposed particle candidates, the most popular one are weakly interacting massive particles (WIMPs) with masses in the range $10 \mathrm{GeV}-10 \mathrm{TeV}$. Although most proposed WIMPs are stable and are produced thermally in the early Universe with an annihilation cross section (times relative velocity) of $\langle\sigma v\rangle \sim 3 \times 10^{-26} \mathrm{~cm}^{3} \mathrm{~s}^{-1}$, DM may be unstable but long-lived, with a lifetime $\tau_{\chi}$ much longer than the age of the Universe $t_{\mathrm{U}} \simeq 4 \times 10^{17} \mathrm{~s}$. Moreover, DM might have been produced via non-thermal processes and have a larger annihilation cross section than the canonical value for WIMP thermal relics.

Among the different ways to detect DM, indirect searches look for the products of DM annihilation or decay, which include antimatter, neutrinos and photons. During the last years, different approaches have been proposed to constrain dark matter properties by using indirect measurements $[1,2,3]$. However, to extract the properties of the DM particle from the detection of an indirect signal requires several pieces of information. There exist many different degeneracies among the different parameters which determine the energy spectrum of the signal. In general, this prevents accurate reconstruction of the DM properties from the energy spectrum alone. In particular, the sole measurement of the energy spectrum would make it impossible to know if the indirect signal from DM is produced by annihilation or decay. The spectrum of the former is characterized by a cutoff at an energy equal to the DM mass, while the cutoff in the spectrum from the latter is at an energy equal to half of the DM mass.

In this talk (see also Ref. [4]), we address the question whether annihilation and/or decay can be identified as the origin of a DM signal in gamma rays. We note that if DM is unstable and produces an observable signal from decay, an annihilation signal will also be present. We show that there is a range of parameters for which the two signals would be comparable, and in this case, angular information could help to determine their presence and their relative contribution to the total signal. Although very challenging, this would identify DM as an unstable particle.

In particular, in order to tackle this problem, we suggest a strategy to distinguish between these scenarios using future gamma-ray observations of Milky Way dwarf galaxies. We show that, in the case that a gamma-ray signal is clearly detected, the origin could be identified as DM decay, annihilation, or both by examining the dependence of the intensity and energy spectrum on the angular distribution of the emission. Furthermore, if annihilation and decay each contribute significantly to the signal, we show how these observations could be used to extract information about the DM mass, lifetime, annihilation cross section, and dominant annihilation and decay channels. In addition, as a byproduct of this analysis, one might also establish or limit the contribution to the signal from substructure in the dwarf galaxy's halo.

\section{General Idea}

An indirect signal from annihilation or decay originates from the same DM particles, but these two processes give rise to different angular distributions of the emission and different energy 
spectra. As pointed out in Refs. [5, 6], angular information is crucial to distinguish DM annihilation from decay. Whereas the rate of DM annihilation scales as the square of the DM density $\rho$, that of DM decay scales linearly with the density, and consequently the angular distribution of the signal from annihilation is expected to follow a steeper profile than that from decay. However the spatial distribution of DM substructure in a halo also scales roughly as $\rho$. Consequently, annihilation in this component could produce a similar flattening in the angular distribution of the observed emission as is expected for decay.

Thus, in order to distinguish these possibilities, we propose an observing strategy based on studying the angular variation of the intensity and the energy spectrum of the signal. From an observational standpoint, a dramatic decrease in the observed intensity between the center of the object and that at larger angles is a clear indicator of the simple case of annihilation in the smooth halo only, while the observation of a shallow emission profile at all angles would strongly suggest decay only. On the other hand, the observation of a bright central region but with the intensity falling off more slowly in the outer regions is less straightforward to interpret, as it could indicate annihilation with an important contribution from substructure, or both annihilation and decay contributing significantly. In this case, we demonstrate that an analysis of the energy spectrum of the signal as a function of angular distance from the center of the object could provide the necessary information to distinguish these possibilities.

If only one process (annihilation or decay) produces a detectable signal, the energy spectrum of the DM signal is the same from all regions of the object, with the intensity varying according to how the rate of that process depends on the DM distribution. If both processes produce detectable signals, the energy spectrum of the total signal varies according to the contribution from each process. With generality, we can assume that in this two-process scenario the annihilation signal is always dominant in the inner regions of the object, with decay becoming more important at larger angles from the center of the object. Thus, we identify that both annihilation and decay are present by observing a change in the energy spectrum of the signal as a function of angle. By measuring this change, the presence of both annihilation and decay is confirmed, so by examination of the signal in the inner and outer regions of the object, the degeneracy in the DM particle mass could be broken. In this case the DM lifetime and annihilation cross section could also be determined from the indirect measurement, up to uncertainties in the density profile and, for the signal from the outer regions, uncertainties in the properties of substructure.

In the following we illustrate the main points just outlined for the case of gamma-ray observations of dwarf galaxies. Dwarf galaxies are extremely DM-dominated, with mass-to-light ratios in the range $100 M_{\odot} / L_{\odot}<M / L<1000 M_{\odot} / L_{\odot}$ [7]. High DM densities coupled with minimal foregrounds due to a scarcity of astrophysical gamma-ray sources make these objects excellent targets for indirect DM searches in gamma-rays. In addition, the predicted emission from DM decay or annihilation in Milky Way dwarfs has a relatively large angular extent ( $\sim$ few degrees), which in principle, makes it possible to map the angular distribution of an observed signal.

We illustrate the proposed technique for three Milky Way dwarf galaxies: Draco, Ursa Minor, and Sagittarius, which are among the most optimistic for detection in gamma-rays (e.g., Refs. [8, 9]), and are all accessible targets with current experiments. We treat separately the contributions from the smooth halo and substructure components to the gamma-ray signal. The smooth halo case alone provides a lower limit on the gamma-ray signal from annihilation for our assumed density 


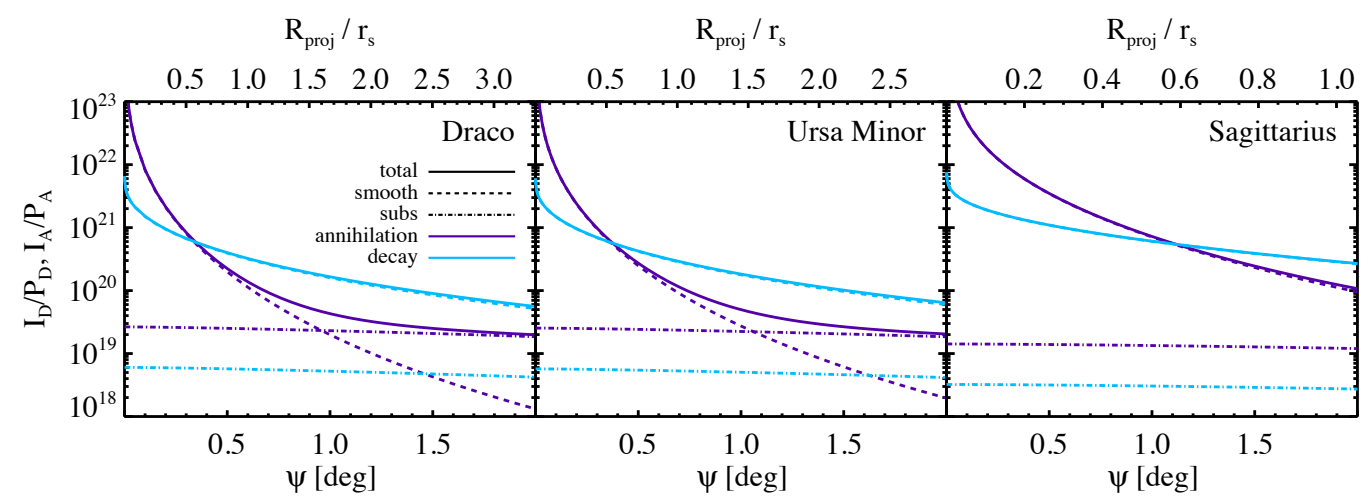

Figure 1: Dependence of the intensity from DM decay (blue) and DM annihilation (purple) on line-of-sight direction $\psi$ from the center of the object for selected dwarf galaxies. The contributions from the smooth halo (dashed), substructure (dot-dashed), and the total (solid) are shown. The corresponding projected radius $R_{\text {proj }}$ in units of the halo scale radius $r_{\mathrm{s}}$ is labeled on the top axis. For generality, the amplitudes of the curves for decay and annihilation have been scaled by the factors $P_{\mathrm{D}}$ and $P_{\mathrm{A}}$ (defined in Ref. [4]) respectively, which depend on the assumed particle properties. $I_{\mathrm{D}} / P_{\mathrm{D}}$ is shown in units of $\mathrm{GeV} \mathrm{cm}{ }^{-2} \mathrm{sr}^{-1}$, and $I_{\mathrm{A}} / P_{\mathrm{A}}$ is shown in units of $\mathrm{GeV}^{2} \mathrm{~cm}^{-5} \mathrm{sr}^{-1}$. From Ref. [4].

profile and represents the steepest angular emission profile. On the other hand, simulations indicate that a scaled-down host subhalo population represents the maximum expected abundance of subsubstructure $[10,11]$, so we model the subhalo population of each dwarf in this way to consider the upper limits on the total annihilation flux and on the shallowness of the angular emission profile in the annihilation case. Let us note however, that the properties of substructure in dwarf galaxy halos are quite uncertain, but for completeness we also consider this potential contribution.

We describe the mass distribution of the smooth DM halo of each dwarf galaxy by a Navarro, Frenk and White (NFW) density profile [12] and the collective emission from subhalos within the dwarf galaxy halo, by summing over the contribution to the gamma-ray signal from subhalos of all masses. We assume that the density profile of each subhalo can also be described by a NFW profile. We refer the reader to Ref. [4] for details on the modeling of the DM distribution and the measured and derived properties of the selected dwarf galaxies.

The angular dependence of the gamma-ray intensity from DM annihilation and decay is shown in Fig. 1 for our three example dwarf galaxies. The contributions from substructure and the smooth halo are shown separately, along with the total of these signals from each process. The contribution from DM annihilation or decay in substructure (blue and purple dot-dashed curves) tends to be nearly parallel to the smooth halo contribution in the case of decay (blue dashed curves) at angles $\gtrsim 1^{\circ}$. Note that decay in substructure is always subdominant relative to decay in the smooth halo, even in the maximal substructure scenario we consider here.

\section{Results}

The first requirement in order to use this strategy is that the source is resolved as an extended source. In particular, we assume that the signal can be binned into several annuli centered on the source. This is in principle possible with the angular resolution of current experiments $\left(\sim 0.1^{\circ}\right.$ at the relevant energies) for observations of dwarf spheroidal galaxies, since the angular extent of the predicted DM signal is as large as $\sim$ few degrees. In addition, this strategy requires that the 


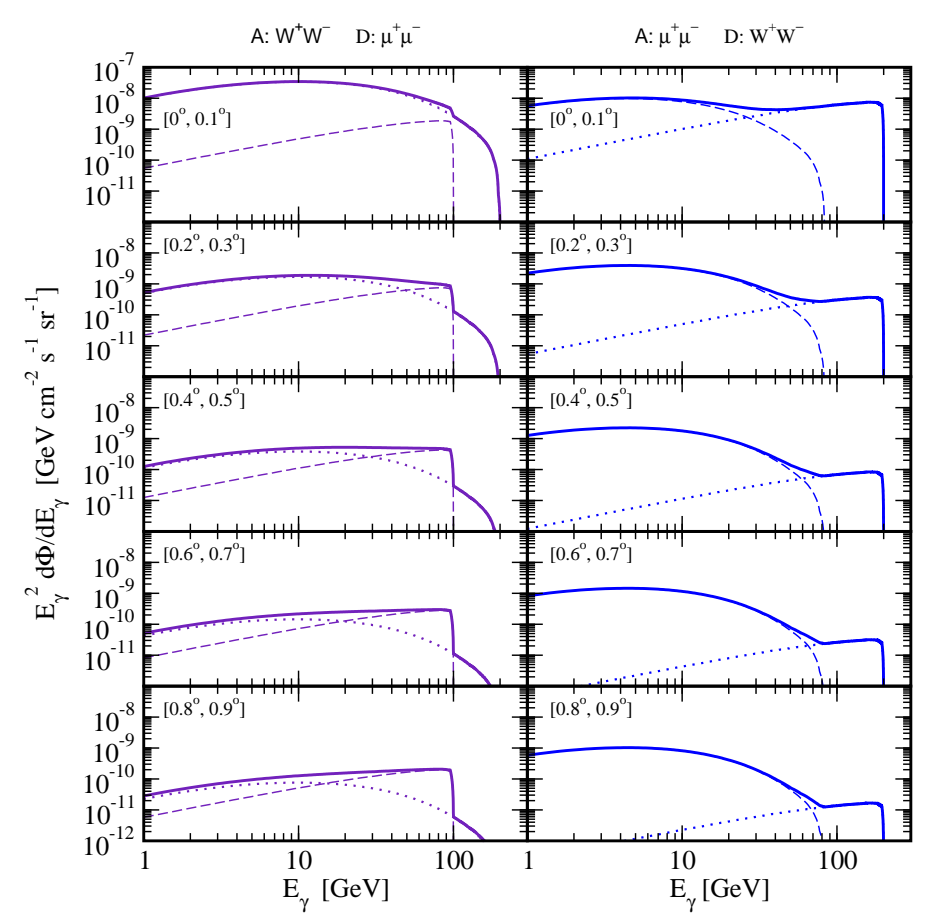

Figure 2: Energy spectra in different annuli centered on Draco for a DM mass of $200 \mathrm{GeV}$ and for two combinations of channels. See text for details. From Ref. [4].

signal in each annulus is detected with sufficient statistics to reconstruct the energy spectrum. In the following we proceed under the assumption that these conditions are met.

In Fig. 2 we illustrate the proposed method for a scenario in which both annihilation and decay contribute appreciably to the observed signal from the Draco dwarf galaxy by showing the energy spectrum as a function of the angle from the center of the object. The energy spectrum in alternating annuli of $0.1^{\circ}$ width centered on Draco is shown out to an angular radius of $0.9^{\circ}$ (from top to bottom) for a DM particle mass of $m_{\chi}=200 \mathrm{GeV}$. Two combinations of channels are shown. The left (right) column shows the case of annihilation into a soft (hard) channel and decay into a hard (soft) one. The channels $\mu^{+} \mu^{-}$and $W^{+} W^{-}$have been chosen as representative of hard and soft channels, respectively. In each panel, dashed lines represent the contribution from decay, dotted lines represent that from annihilation, and the thick solid lines represent the total contribution. We have taken $\langle\sigma v\rangle=3 \times 10^{-26} \mathrm{~cm}^{3} \mathrm{~s}^{-1}$ and $\tau_{\chi}=10^{29} \mathrm{~s}$. Note that although we have included the contribution of substructure, it is a subdominant effect for both annihilation and decay for the annuli considered in this figure (see Fig. 1). As expected, a significant change in the spectrum is clearly seen in Fig. 2 for both combinations of channels at around $E=m_{\chi} / 2$, i.e., the maximum energy for photons from DM decay. The spectral change is a signature of both annihilation and decay contributing significantly to the signal.

Fig. 3 indicates the range of DM parameters which would induce a transition between annihilation and decay in the angular range of $0^{\circ}-2^{\circ}$ in Draco, (similar results are obtained for the other two dwarf galaxies). Here we neglect the contribution from substructure. The curves indicate the value of the DM lifetime at which the intensities from DM annihilation and decay integrated above $1 \mathrm{GeV}$ 

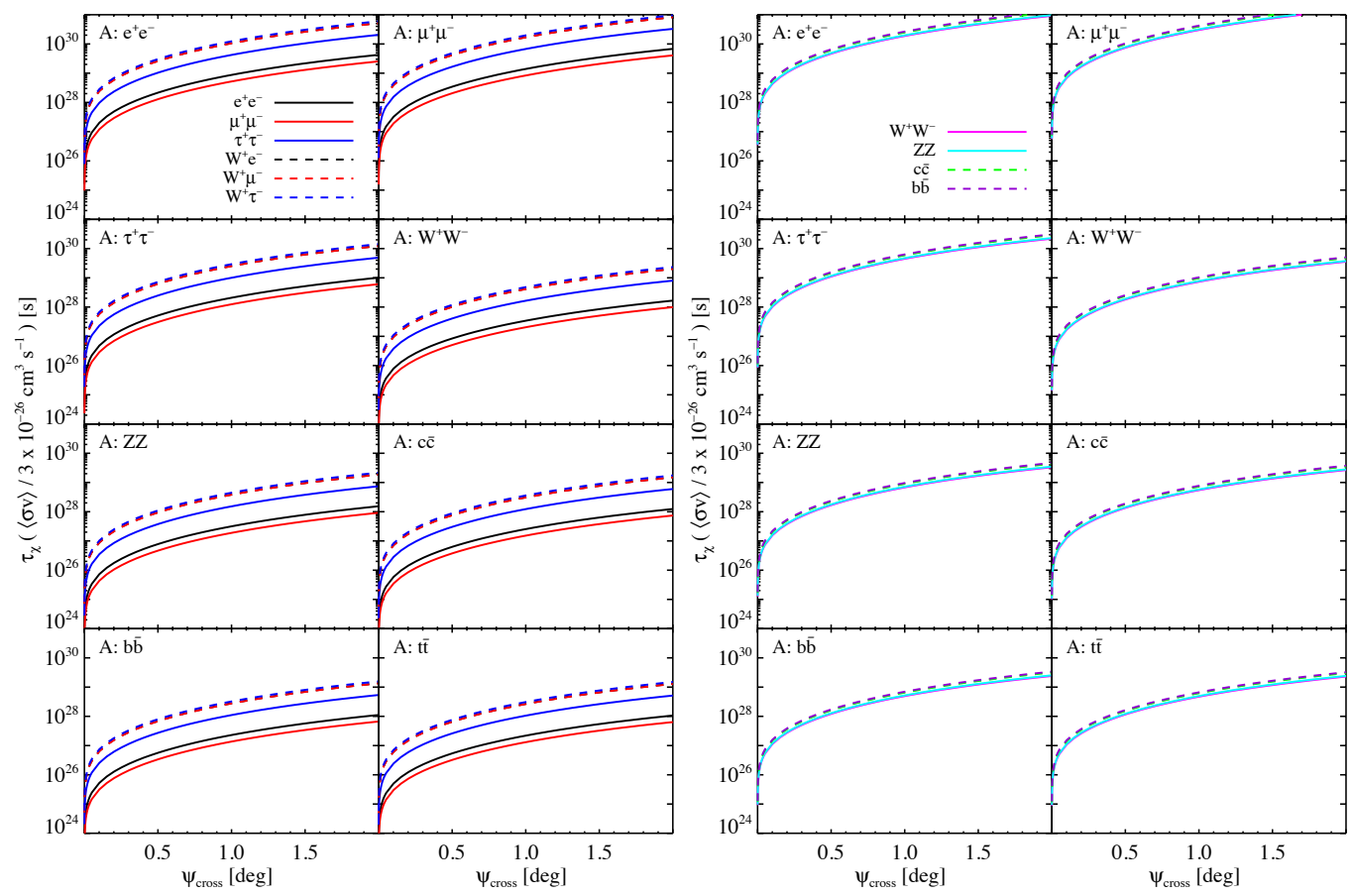

Figure 3: Lifetime $\tau_{\chi}$ for $m_{\chi}=200 \mathrm{GeV}$ at which the intensities from annihilation and decay for $E>1 \mathrm{GeV}$ are equal at an observation angle $\psi_{\text {cross }}$ from the center of the dwarf galaxy, for Draco, without substructure. Each panel shows curves for a single annihilation channel, assuming decay into different channels (as labeled). From Ref. [4].

are equal at an observation angle $\psi_{\text {cross. }}$. The results for DM decay into leptonic and semileptonic (hadronic and gauge boson) channels are shown in the left (right) panels. The annihilation channel for each panel is labeled. In these figures we assume $m_{\chi}=200 \mathrm{GeV}$ and $\langle\sigma v\rangle=3 \times 10^{-26} \mathrm{~cm}^{3} \mathrm{~s}^{-1}$. A larger cross section would displace the curves downwards. For a given $\psi_{\text {cross }}$, above the curves annihilation dominates and the emission profile is steeper, while below the curves the dominant contributor is decay and the profile is shallower.

The normalization of the curves depends on the relative photon yields from annihilation and decay: for a given lifetime, the annihilation-to-decay transition occurs further from the center of the dwarf galaxy for channel combinations in which the ratio of the photon yields from annihilation to decay is larger. In each panel, corresponding to a single annihilation channel, the variation in the amplitude of the curves reflects the different photon yields for the decay channels shown. Decay via any of the hadronic or gauge boson channels produces almost identical curves since the photon yields above $1 \mathrm{GeV}$ from these channels are similar, and these curves have the highest normalization of any of the channels since their photon yields are the highest. Similarly, there is little difference between the curves for decay into any of the three semi-leptonic channels, and these curves fall below the hadronic and gauge boson decay channel curves. The curves for decay into the leptonic channels show more variation due to the larger variation in photon yields for these channels, and as expected, fall below those for semi-leptonic and hadronic and gauge boson channels due to their relatively low photon yields.

For this energy threshold and an assumed cross section of $\langle\sigma v\rangle=3 \times 10^{-26} \mathrm{~cm}^{3} \mathrm{~s}^{-1}$, in order for the transition to occur at an angle between $\sim 0.1^{\circ}$ and $\sim 2^{\circ}$, the DM lifetime must be between 
$\sim 10^{25} \mathrm{~s}$ and $10^{31} \mathrm{~s}$, depending on the combination of channels. For larger values of the annihilation cross section, correspondingly smaller values of the lifetime are needed.

\section{Conclusions}

In this talk we have outlined a strategy to constrain DM properties in the event of the clear detection of an indirect signal from gamma-ray observations of dwarf galaxies. We addressed the question of how scenarios of DM annihilation, decay, or both could be distinguished, and what information could be obtained about the intrinsic properties of the DM particle and its small-scale distribution from this type of indirect measurement. In summary, we have shown that a DM particle with an annihilation cross-section and lifetime just beyond the limits currently established could produce a clear spectral change on an angular scale within the reach of future experiments. Ongoing observations by current and future experiments will continue to improve the prospects for detecting and mapping a DM signal in the coming years.

\section{References}

[1] J. Edsjo and P. Gondolo, Phys. Lett. B 357, 595 (1995) [arXiv:hep-ph/9504283]; M. Cirelli, N. Fornengo, T. Montaruli, I. Sokalski, A. Strumia and F. Vissani, Nucl. Phys. B 727, 99 (2005) [Erratum-ibid. B 790, 338 (2008)] [arXiv:hep-ph/0506298]; O. Mena, S. Palomares-Ruiz and S. Pascoli, Phys. Lett. B 664, 92 (2008) [arXiv:0706.3909 [hep-ph]].

[2] S. Dodelson, D. Hooper and P. D. Serpico, Phys. Rev. D 77, 063512 (2008) [arXiv:0711.4621 [astro-ph]]; N. Bernal, A. Goudelis, Y. Mambrini and C. Muñoz, JCAP 0901, 046 (2009) [arXiv:0804.1976 [hep-ph]]; T. E. Jeltema and S. Profumo, JCAP 0811, 003 (2008) [arXiv:0808.2641 [astro-ph]]; N. Bernal and S. Palomares-Ruiz, arXiv:1006.0477 [astro-ph.HE].

[3] B. S. Hensley, J. M. Siegal-Gaskins and V. Pavlidou, Astrophys. J. 723, 277 (2010) [arXiv:0912.1854 [astro-ph.CO]].

[4] S. Palomares-Ruiz and J. M. Siegal-Gaskins, JCAP 1007, 023 (2010) [arXiv:1003.1142 [astro-ph.CO]].

[5] G. Bertone, W. Buchmuller, L. Covi and A. Ibarra, JCAP 0711, 003 (2007) [arXiv:0709.2299 [astro-ph]].

[6] S. Palomares-Ruiz, Phys. Lett. B 665, 50 (2008) [arXiv:0712.1937 [astro-ph]].

[7] J. D. Simon and M. Geha, Astrophys. J. 670, 313 (2007) [arXiv:0706.0516 [astro-ph]]; N. F. Martin, J. T. A. de Jong and H. W. A. Rix, Astrophys. J. 684, 1075 (2008) [arXiv:0805.2945 [astro-ph]].

[8] R. Essig, N. Sehgal and L. E. Strigari, Phys. Rev. D 80, 023506 (2009) [arXiv:0902.4750 [hep-ph]].

[9] A. A. Abdo et al. [Fermi LAT Collaboration], arXiv:1001.4531 [astro-ph.CO].

[10] J. Diemand, M. Kuhlen, P. Madau, M. Zemp, B. Moore, D. Potter and J. Stadel, Nature 454, 735 (2008). [arXiv:0805.1244 [astro-ph]].

[11] V. Springel et al., Mon. Not. Roy. Astron. Soc. 391, 1685 (2008) [arXiv:0809.0898 [astro-ph]].

[12] J. F. Navarro, C. S. Frenk and S. D. M. White, Mon. Not. Roy. Astron. Soc. 275, 720 (1995) [arXiv:astro-ph/9408069]; J. F. Navarro, C. S. Frenk and S. D. M. White, Astrophys. J. 462, 563 (1996) [arXiv:astro-ph/9508025]; J. F. Navarro, C. S. Frenk and S. D. M. White, Astrophys. J. 490, 493 (1997) [arXiv:astro-ph/9611107]. 